\title{
Efficient full waveform inversion using the excitation representation of the source wavefield
}

\author{
Mahesh Kalita and Tariq Alkhalifah \\ Department of Physical Science and Engineering, King Abdullah University of Science and Technology, Thuwal 23955-6900, Saudi Arabia. \\ E-mail:mahesh.kalita.2@kaust.edu.sa
}

Accepted 2017 May 16. Received 2017 May 13; in original form 2016 September 20

\begin{abstract}
S U M MAR Y
Full waveform inversion (FWI) is an iterative method of data-fitting, aiming at high-resolution recovery of the unknown model parameters. However, its conventional implementation is a cumbersome process, requiring a long computational time and large memory space/disk storage. One of the reasons for this computational limitation is the gradient calculation step. Based on the adjoint state method, it involves the temporal cross-correlation of the forward propagated source wavefield with the backward propagated residuals, in which we usually need to store the source wavefield, or include an extra extrapolation step to propagate the source wavefield from its storage at the boundary. We propose, alternatively, an amplitude excitation gradient calculation based on the excitation imaging condition concept that represents the source wavefield history by a single, specifically the most energetic arrival. An excitation based Born modelling allows us to derive the adjoint operation. In this case, the source wavelet is injected by a cross-correlation step applied to the data residual directly. Representing the source wavefield through the excitation amplitude and time, we reduce the large requirements for both storage and the computational time. We demonstrate the application of this approach on a two-layer model with an anomaly, the Marmousi II model and a marine data set acquired by CGG.
\end{abstract}

Key words: Inverse theory; Tomography; Seismic tomography.

\section{INTRODUCTION}

Although seismic full waveform inversion (FWI) is a powerful tool to estimate the unknown subsurface parameters quantitatively with high resolution (Lailly 1983; Tarantola 1984; Gauthier et al. 1986; Pratt 1990; Pratt et al. 1996), it is computationally very expensive. The cost of FWI in the framework of local nonlinear-optimization problem is incurred mostly by the computation of the gradient at each iteration. The choice of optimization methods in the inversion process realm (Pratt et al. 1998; Tarantola 2005; Nocedal \& Wright 2006; Ma \& Hale 2012; Métivier et al. 2014) governs the number of iterations required to achieve a converging model. Despite huge improvements in the computational power and algorithm, it can be a challenge to make FWI efficient, especially in large-scale 3-D problems (Virieux \& Operto 2009).

Over the past years, numerous solutions have been proposed to mitigate the well-known problem of non-linearity in FWI. The most popular one is the multiscale strategy (Bunks et al. 1995; Sirgue \& Pratt 2004; Boonyasiriwat et al. 2009) in which we gradually append the high-frequency details of the data to the inversion result of low frequency. Alternatively, Biondi \& Almomin (2014) successfully inverts the full bandwidth of the data. Recently, the merger of migration velocity analysis with FWI (Symes 2008; Almomin
\& Biondi 2012; Allemand \& Lambaré 2015; Wu \& Alkhalifah 2015b; Alkhalifah \& Wu 2016) has gained popularity in the context of improving the prospect of converging to a global minimum. Moreover, the isolation of desired wavenumbers in the gradient using conditioning and filtering (Albertin et al. 2013; Tang et al. 2013; Alkhalifah 2015; Kazei et al. 2016) helps such convergence prospect. Despite the potential of all these methods to alleviate the non-linearity issue, they usually pay the price with a significant increase in computational cost.

FWI iteratively updates the model parameters with a quantity that depends on the gradient of the misfit between the observed and the simulated seismic data. No extra computation of the huge Jacobian matrix is required since the gradient is equivalent to the image in reverse time migration (RTM) process (Lailly 1983; Plessix 2006). We, specifically, need three key steps to compute the gradient in a time domain formulation. Step one is extrapolation of the source wavefield forward in time; step two is extrapolation of the adjoint source backward in time and step three is application of an imaging condition like operation. Since, FWI usually requires a large number of iterations for convergence, the modelling operator and the imaging criteria should be cheap to make it efficient. On the other hand, the need for more sophisticated algorithms incorporating all kinds of physics (anisotropy, elasticity, viscosity) results in 
extra computational costs in FWI. However, source-encoding schemes (Krebs et al. 2009; Choi \& Alkhalifah 2011; Schuster et al. 2011) have drastically reduced the computational cost, albeit with a trade-off in the accuracy of physics.

One of the main reasons for the cost in FWI as mentioned earlier is the gradient calculation step which involves expensive temporal cross-correlation. It requires an order of $n t$ (time samples in a trace) floating point operations (FLOPs) at the time of the correlation for every model point. Additionally, depending on the size of the wavefield, defined by the model and data, a large memory space/disk storage is required in order to store the history of the source wavefield. The boundary saving scheme is a solution (Berkhout 1988; Clapp 2008), but at the cost of an additional wavefield extrapolation. Also, techniques based on wavefield compression either temporally or spatially or both are viable alternate solutions maintaining a balance between computational overhead and time (Unat et al. 2009; Dalmau et al. 2014; Boehm et al. 2016). Although promising, these kind of techniques are also not a panacea in complex models, involving trade-offs in the degree of compression and the amount of distortion while decompressing (Mittal \& Vetter 2016). In this paper, we employ the excitation imaging condition concept in the context of FWI, which helps mitigate the memory issue in an efficient manner. It considers the most energetic arrival of the source wavefield (Chang \& McMechan 1986; Nguyen \& McMechan 2013), resulting in the reduction of the FLOPs during the imaging process. Additionally, we focus on investigating the forward problem using the excitation method.

The global seismic tomography effort for a detailed regional structure of the Earth's interior requires fine numerical grids in the model. As a consequence of Courant-Friedrichs-Lewy (CFL) condition, we have to march the wavefield using small time increments. Also to capture the fine-scale heterogeneities, the inversion process tends to exploit the entire recorded seismogram as much as possible, rather than any isolated part of it. These factors lay down computational challenges of accessing large memory and investing long time in large scale global seismic tomography (Fichtner et al. 2013). The excitation method in this regard is promising as it reduces the computational requirements to a feasible level. Since the principle objective of this article is to develop the methodology of excitation approach in the inversion realm, we provide only the examples for acoustic FWI. Detailed discussions on elastic and anisotropic inversion will be reported in follow up papers. The outline of the paper is as follows. In the theory section, we first define the excitation approximation (ExA). Next, we introduce the scattering problem under ExA. Also, we carry out two numerical examples pertaining to the first-order approximation of scattering theory. Next, we focus on investigating the inverse problem formulation using ExA. Lastly, we demonstrate its versatility in conjunction with FWI on a two-layer model with an anomaly, the Marmousi II model and a real data set acquired offshore Australia by CGG.

\section{THEORY}

The ExA represents the source wavefield by its peak amplitude and arrival time, referred to as excitation amplitude and excitation time $\left(t_{\mathrm{ex}}\right)$, respectively. It assumes that all the arrivals other than excitation amplitude are very small and can be ignored safely. Mathematically, the excitation wavefield $G_{0 \mathrm{ex}}$ at an image point $\mathbf{x}$ due to a source positioned at $\mathbf{x}=\mathbf{x}_{s}$, is a scaled impulse defined as

$$
\begin{aligned}
G_{0_{\mathrm{ex}}}\left(\mathbf{x}, t ; \mathbf{x}_{s}\right) & =G_{0}\left(\mathbf{x}, t ; \mathbf{x}_{s}\right) \delta\left(t-t_{\mathrm{ex}}\left(\mathbf{x} ; \mathbf{x}_{s}\right)\right) \\
& = \begin{cases}G_{0}\left(\mathbf{x}, t_{\mathrm{ex}} ; \mathbf{x}_{s}\right) & \text { for } t=t_{\mathrm{ex}} ; \\
0 & \text { otherwise; }\end{cases}
\end{aligned}
$$

where $G_{0}$ is the Green's function. The subscript ex stands for the excitation representation. This strong assumption is possible primarily because of the simple nature of the source wavelet. However, it draws two important limitations in the context of FWI:

(i) Its simple implementation does not account for multipathing of the source wavefield (Nguyen \& McMechan 2013).

(ii) In addition, eq. (1) does not take into account the source signature, which limits the basin of attraction in the inversion process. However, convolution of source-wavelet, $f(t)$ with $G_{0_{\mathrm{ex}}}$ retrieves its signature. Mathematically, the excitation source wavefield embedded with the source signature, can be defined as

$$
\begin{aligned}
\tilde{G}_{0_{\mathrm{ex}}}\left(\mathbf{x}, t ; \mathbf{x}_{s}\right) & =G_{0_{\mathrm{ex}}} \star f(t) \\
& =G_{0}\left(\mathbf{x}, t ; \mathbf{x}_{s}\right) \delta\left(t-t_{\mathrm{ex}}\left(\mathbf{x} ; \mathbf{x}_{s}\right)\right) \star f(t),
\end{aligned}
$$

where $\star$ represents a convolution process in time. Therefore, ExA in FWI replaces the source wavefield by a modified source function wavelet where its amplitude is scaled by the excitation amplitude and time of origin is shifted by the excitation time. It helps reconstruct the source wavefield partially with a limitation in propagation effects. Mainly, it is insensitive to the dynamic variation in wavelength and the shape of the source wavefield, which tends to agree with our high frequency asymptotic approximation. Thanks to the modification in the adjoint source as described later, this limitation does not cause any issues in the gradient computation procedure of FWI.

To illustrate ExA, we compare the wavefield and its excitation representation at a model point in a homogeneous velocity model shown in Fig. 1. Fig. 1(a) displays the velocity model $\left(v_{0}=2.0 \mathrm{~km}\right.$ $\mathrm{s}^{-1}$ ) whereas the top and bottom panels in Fig. 1(b) represent the source function (Ricker wavelet) at $(x, z)=(1.0,0) \mathrm{km}$ and wavefield at $(x, z)=(1.0,0.5) \mathrm{km}$, respectively. Also, the bottom panel marks the excitation time and amplitude of the source wavefield. Moreover, we overlay the excitation wavefield after retrieving the source signature by a time convolution process as shown in eq. (2). However, we observe a serious disagreement in the maximum energy arrival time of the full wavefield and its corresponding excitation wavefield. This mismatch arises due to the causal and continuous nature of the source wavelet, maintaining a constant lag between the onset and excitation time of the source wavefield throughout its extrapolation. Subtracting the lag from the excitation time rectifies the time shift issue as shown in the next section.

\subsection{The forward problem: Born modelling with ExA}

The Born approximation establishes a linear relationship between scattered wavefield $(\delta G)$ and its cause (model perturbation, $\delta v$ ). Mathematically, $\delta G$ recorded at a receiver position $\mathbf{x}=\mathbf{x}_{r}$ is a temporal convolution process of two Green's functions, one being the second-order temporal differentiation of the source wavefield and another one the receiver wavefield to the point of perturbation:

$$
\begin{aligned}
\delta G\left(\mathbf{x}_{r}, t, \mathbf{x}_{s}\right)= & \int \mathrm{d} \mathbf{x} \frac{\delta v(\mathbf{x})}{v_{0}^{2}(\mathbf{x})} \int \mathrm{d} \tau \frac{\partial^{2}}{\partial t^{2}}\left\{G_{0}\left(\mathbf{x}, t-\tau ; \mathbf{x}_{s}\right)\right\} \\
& \times G_{0}\left(\mathbf{x}_{r}, \tau ; \mathbf{x}\right),
\end{aligned}
$$




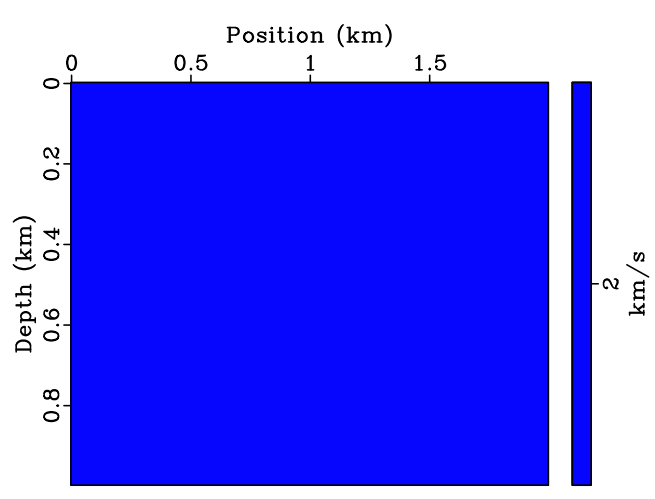

(a)
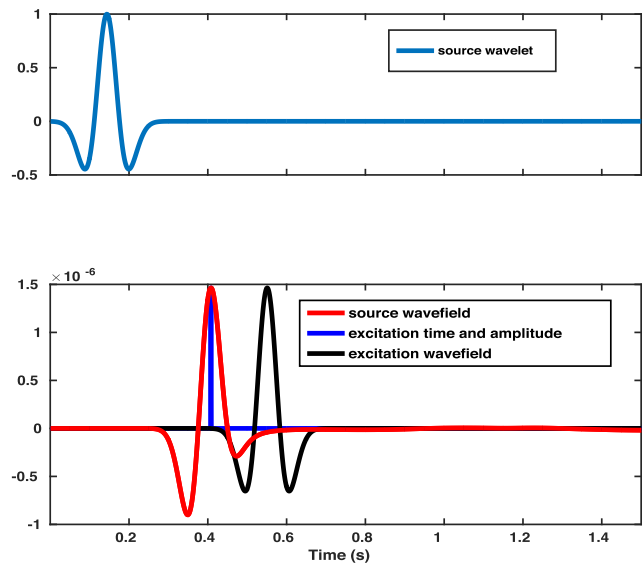

(b)

Figure 1. (a) Homogeneous velocity model $\left(v_{0}=2.0 \mathrm{~km} \mathrm{~s}^{-1}\right)$; (b) top panel depicts source wavelet; bottom panel compares the full wavefield, its excitation time and amplitude and the excitation wavefield embedded with the source signature at a model point $(x, z)=(1.0,0.5) \mathrm{km}$.

with $\delta v$ satisfying $\frac{1}{v^{2}}=\frac{1}{v_{0}^{2}}(1+\delta v)$, where $v$ and $v_{0}$ are the true and reference model, respectively. Now, eq. (2) in eq. (3) yields the excitation representation of $\delta G$, given by

$$
\begin{aligned}
\delta G_{\mathrm{ex}}\left(\mathbf{x}_{r}, t, \mathbf{x}_{s}\right)= & \int \mathrm{d} \mathbf{x} \frac{\delta v(\mathbf{x})}{v_{0}^{2}(\mathbf{x})} \int \mathrm{d} \tau \frac{\partial^{2}}{\partial t^{2}}\left\{\tilde{G}_{0_{\mathrm{ex}}}\left(\mathbf{x}, t-\tau ; \mathbf{x}_{s}\right)\right\} \\
& \times G_{0}\left(\mathbf{x}_{r}, \tau ; \mathbf{x}\right) .
\end{aligned}
$$

Therefore, the scattered data using Born and ExA satisfies the following wave equation:

$$
\begin{aligned}
& \left(\nabla^{2}-\frac{1}{v_{0}^{2}} \frac{\partial^{2}}{\partial t^{2}}\right) \delta G_{\mathrm{ex}}\left(\mathbf{x}_{r}, t ; \mathbf{x}_{s}\right) \\
& =\frac{\delta v(\mathbf{x})}{v_{0}^{2}(\mathbf{x})} \frac{\partial^{2}}{\partial t^{2}}\left\{\tilde{G}_{0_{\mathrm{ex}}}\left(\mathbf{x}, t ; \mathbf{x}_{s}\right)\right\} \\
& =\frac{\delta v(\mathbf{x})}{v_{0}^{2}(\mathbf{x})} G_{0}\left(\mathbf{x}, t_{\mathrm{ex}} ; \mathbf{x}_{s}\right) \frac{\partial^{2}}{\partial t^{2}}\left\{f\left(t-t_{\mathrm{ex}}\right)\right\} .
\end{aligned}
$$

Similar to the conventional Born modelling, implementation of excitation method requires two for loops: the first one computes the excitation time and amplitude to the point of perturbation and the second one solves eq. (5). However, it yields a subtle time-shift in the recorded scattered data compared to the conventional approach because of the reason mentioned in the last section. However, that lag can be alleviated by simply subtracting it from the excitation time. Next, we illustrate the Born modelling based on the excitation approach with the two following examples:

(i) Single scatterer in a homogeneous model. We display the scattered data (Fig. 2) induced by a single point perturbation in a homogeneous velocity model of Fig. 1(a). The difference of the data modelled with the actual velocity $\left(v=v_{0}+\delta v\right)$ and the background velocity $\left(v_{0}\right)$ is shown in Fig. 2(b). As expected, the born modelling gives rise to a traveltime error (Fig. 2c). Thus, the excitation Born modelling can accurately reproduce the single scattered wavefield (Fig. 2d), albeit with a correction in the traveltime.

(ii) Blocks of scatterers in Marmousi model. This example compares the scattered data (Fig. 3d) induced because of two blocks of scatterers (Fig. 3b) in the Marmousi model (Fig. 3a), with the residual between the true and reference data sets (Fig. 3c). The Born scattered data with the excitation approach, although being the first order approximation to the Lippmann Schwinger equation, is comparable with the data residual, which is computed using a finite-difference scheme.

\subsection{The inverse problem: FWI with ExA}

The gradient of FWI, based on the $L^{2}$ norm misfit function is given by:

$g(\mathbf{x})=\frac{-2}{v_{0}^{3}(\mathbf{x})}\left[\frac{\partial^{2} \vec{G}_{0}\left(\mathbf{x}, t ; \mathbf{x}_{s}\right)}{\partial t^{2}} \bullet \overleftarrow{\Delta d}\left(\mathbf{x}, t ; \mathbf{x}_{s}\right)\right]$

where $\Delta d$ is the data residual between the predicted $\left(d_{m}\right)$ and the observed $\left(d_{o}\right)$ data, with $\bullet$ represents the zero-lag temporal crosscorrelation process and $\overrightarrow{()}, \overleftarrow{()}$ represent the forward and backward propagation of wavefields in time, respectively. Now, eq. (6) using the ExA becomes

$$
\begin{aligned}
g_{\mathrm{ex}}(\mathbf{x}) & =\frac{-2}{v_{0}^{3}(\mathbf{x})}\left[\left\{G_{0}\left(\mathbf{x}, t ; \mathbf{x}_{s}\right) \delta\left(t-t_{\mathrm{ex}}\right) \star \frac{\partial^{2} f(t)}{\partial t^{2}}\right\} \bullet \overleftarrow{\Delta d}\left(\mathbf{x}, t ; \mathbf{x}_{s}\right)\right] \\
& =\frac{-2}{v_{0}^{3}(\mathbf{x})} G_{0}\left(\mathbf{x}, t_{\mathrm{ex}} ; \mathbf{x}_{s}\right)\left[\frac{\partial^{2} f\left(t-t_{\mathrm{ex}}\right)}{\partial t^{2}} \bullet \overleftarrow{\Delta d}\left(\mathbf{x}, t ; \mathbf{x}_{s}\right)\right] .
\end{aligned}
$$

Based on this formula, the needed information from the source wavefield history is only the maximum amplitude and its arrival time in order to compute the gradient. Thus, this results in significant saving of memory space/disk-storage. Also, because of the source wavelet, the gradient now involves a very small window for crosscorrelation, ensuring a very few FLOPs at the time of imaging (or other words, replaces the dot product with a selection process). Consequently, this method leads to a reduction in computational cost in comparison to its conventional counterpart.

We can formulate the problem in matrix form as follows:

$g_{\text {ex }}(\mathbf{x})=\left\langle\mathbf{F} \mathbf{u}_{\delta}, \mathbf{L}^{*} \Delta d\right\rangle$,

where $\langle$,$\rangle represents the dot product between the two vectors.$ $\mathbf{F}$ is a lower triangular Toeplitz matrix with $j$ th row given by $\left(\left.\left.\left.\frac{\partial^{2} f}{\partial t^{2}}\right|_{t_{j}} \ldots \frac{\partial^{2} f}{\partial t^{2}}\right|_{t_{2}} \frac{\partial^{2} f}{\partial t^{2}}\right|_{t_{1}} \ldots 0\right)$ that acts on the spike source wavelet $u_{\delta}$ with non-zero element at the excitation time $t_{\text {ex }}$, representing the causal convolution process. $\mathbf{L}^{*}$ is the adjoint of the wave equation operator, signifying the reverse extrapolation of the adjoint source in time. Using the properties of the dot product, we can rewrite eq. (8) as

$g_{\mathrm{ex}}(\mathbf{x})=\left\langle\mathbf{u}_{\delta}, \mathbf{F}^{*} \mathbf{L}^{*} \Delta d\right\rangle$ 


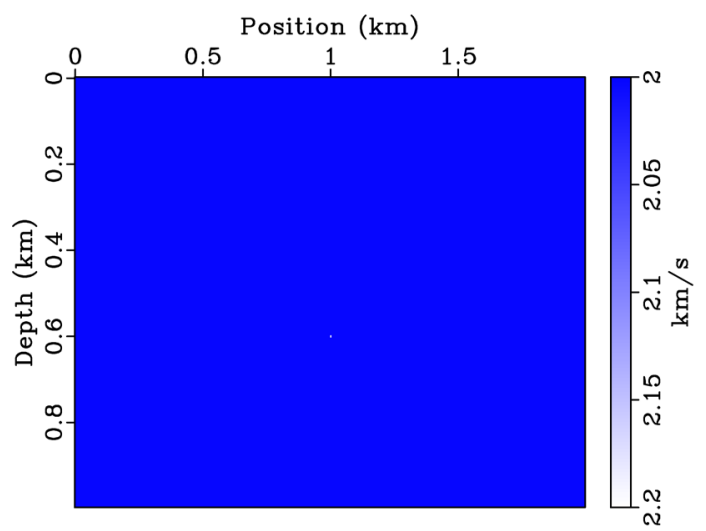

(a)

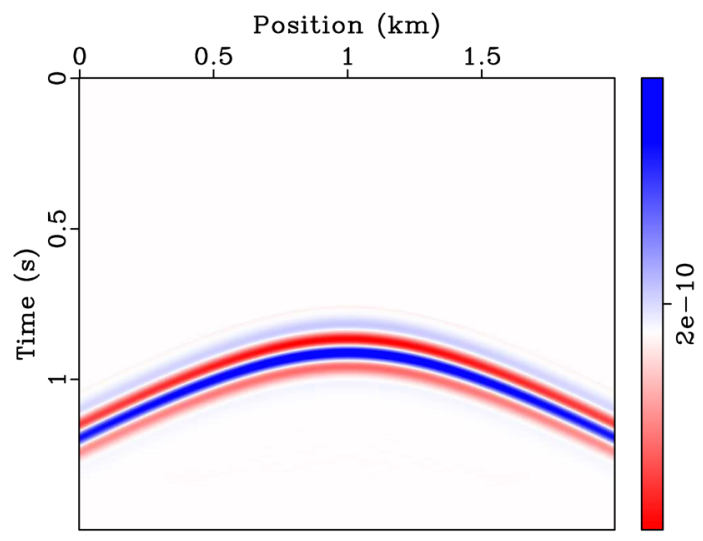

(c)

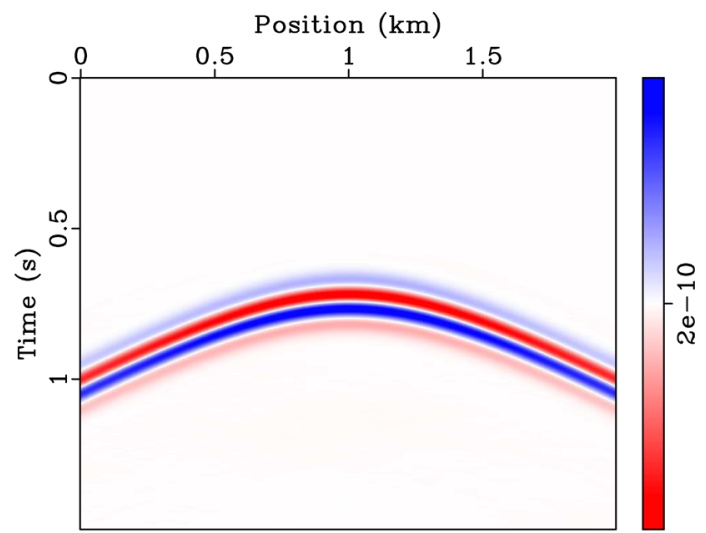

(b)

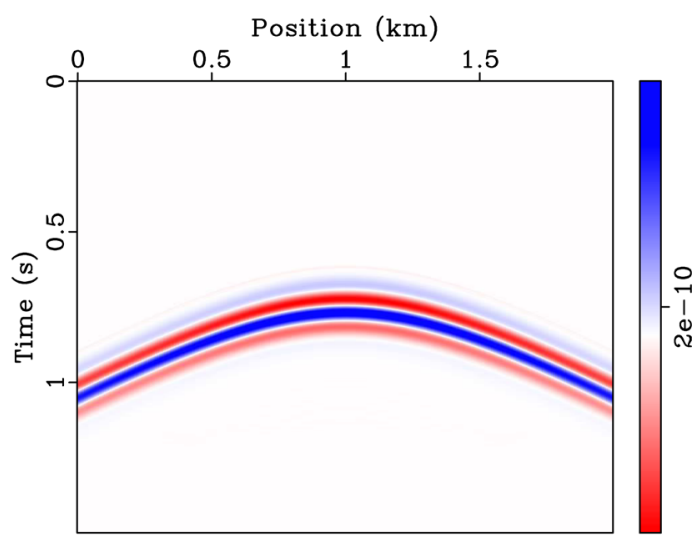

(d)

Figure 2. (a) Velocity model $v$; (b) data modelled with actual velocity $v$ - data modelled with velocity $v_{0}=2.0 \mathrm{~km} \mathrm{~s}^{-1}$; (c) shifted scattered data using excitation method; (d) after the correction in panel (c).

where $\mathbf{F}^{*}$ is the cross-correlation operator. Since the solution of the wave equation is linear with respect to its source, we can interchange the positions of the operators $\mathbf{F}^{*}$ and $\mathbf{L}^{*}$. Therefore, eq. (9) becomes

$g_{\text {ex }}(\mathbf{x})=\left\langle\mathbf{u}_{\delta}, \mathbf{L}^{*} \mathbf{F}^{*} \Delta d\right\rangle$.

It suggests that we need to cross-correlate the second derivative of the source function $f$ with $\Delta d$ before the reverse extrapolation. Since $u_{\delta}$ contains only one non-zero element given by $-\frac{G_{0}\left(\mathbf{x}, t_{\mathrm{ex}} ; \mathbf{x}_{\mathrm{s}}\right)}{v_{0}^{3}(\mathbf{x})}$, the imaging process requires the adjoint wavefield only at the time of origin $\left(t_{\mathrm{ex}}\right)$, and thus, a single FLOP. Therefore, the computation of gradient using the excitation approach is given by

$g_{\mathrm{ex}}(\mathbf{x})=\mathbf{u}_{\delta}\left(\mathbf{x}, t_{\mathrm{ex}}(\mathbf{x})\right) \mathbf{v}\left(\mathbf{x}, t_{\mathrm{ex}}(\mathbf{x})\right)$ with
$\mathbf{v}=\mathbf{L}^{*} \Delta \hat{d}$ and $\Delta \hat{d}=\mathbf{F}^{*} \Delta d$,

where $\Delta \hat{d}$ and $\mathbf{v}$ represent the adjoint source and wavefield, respectively.

\subsection{Sensitivity kernel}

In seismic waveform tomography, the sensitivity kernel or the Fréchet derivative suggests the shape of the Fresnel zone along the wave path (Woodward 1992; Tromp et al. 2005; van der Hilst \& de Hoop 2005; Djebbi \& Alkhalifah 2013) in order to delineate the Earth model in local and global scale. Therefore, it provides insights into the possible model perturbations necessary to explain an event in the data, or the residuals between modelled and observed data. In other words, it describes all the possible areas in the model that are capable of influencing the data, for example, at a certain receiver. We, thus, compare the kernel produced by ExA with that by its conventional counterpart. We consider a velocity model linearly increasing with depth as shown in Fig. 4(a). A source and receiver are placed at $(x, z)=(2.0,0.5) \mathrm{km}$ and $(x, z)=(12.0$, $0.5) \mathrm{km}$, respectively. As expected, the banana kernel using ExA is generally similar to that of the conventional, but produced with less computational resources (Figs $4 \mathrm{~b}$ and $\mathrm{c}$ ). We display a depth profile at $x=7.0$ in Fig. 4(d) which suggests the kinematic equivalence between the two methods.

\subsection{Pseudo-Hessian with ExA}

Shin et al. (2001) demonstrated that the diagonal of the pseudoHessian plays a vital role in compensating for the geometrical spreading loss by scaling the gradient with a proper weight. In the excitation approach, the scaling is simply an arithmetic division of the gradient by the square of the excitation amplitude. In order to strictly obey the ExA, we prefer the divisor to be excitation amplitude squared, rather than the source normalized term $\left(\sum_{i t=1}^{n t} G\left(\mathbf{x}, i t ; \mathbf{x}_{s}\right)^{2}\right)$ despite that both shares almost the same computational cost. Finally, the model update $\tilde{g}_{\text {ex }}$ at an iteration is given 


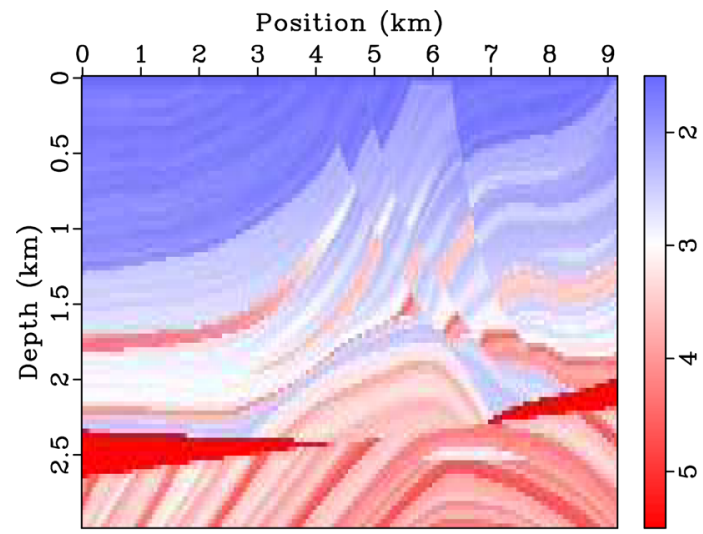

(a)

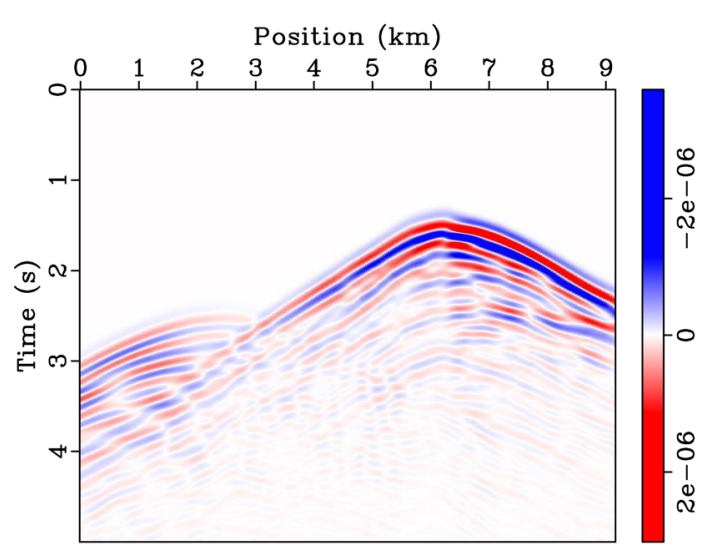

(c)

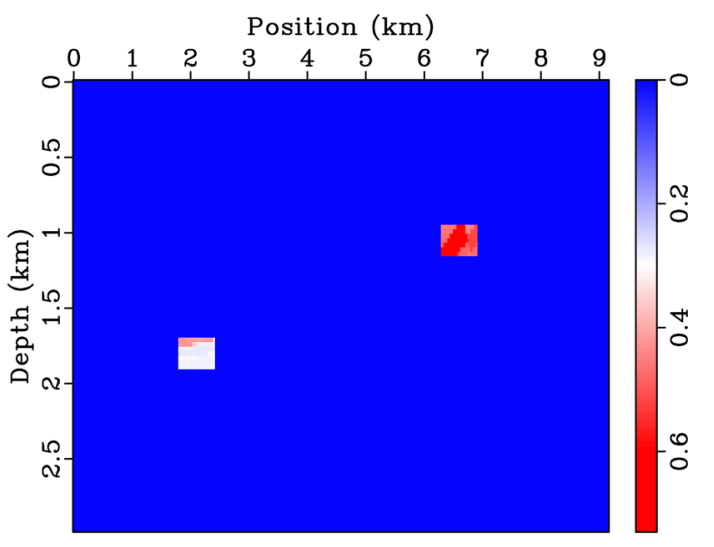

(b)

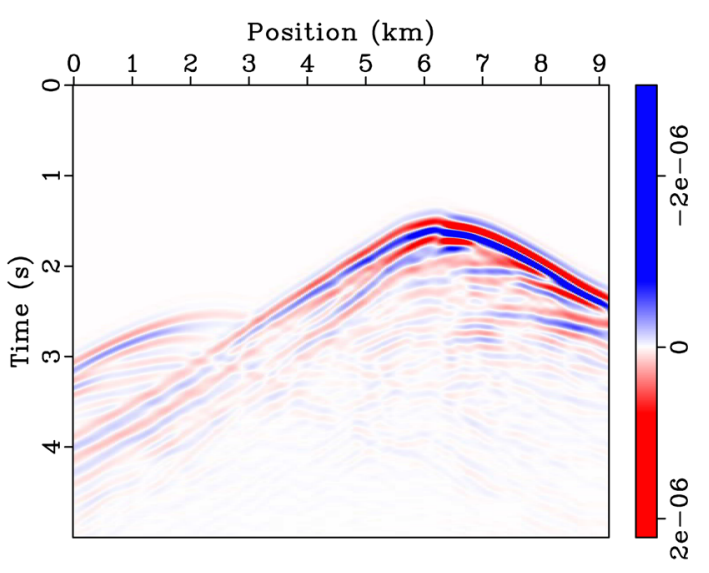

(d)

Figure 3. (a) Marmousi model $\left(v_{0}\right)$; (b) scattering blocks $(\delta v)$; (c) data modelled with $\left(v_{0}+\delta v\right)$ - data modelled with $v_{0}$; (d) scattered data using excitation method.

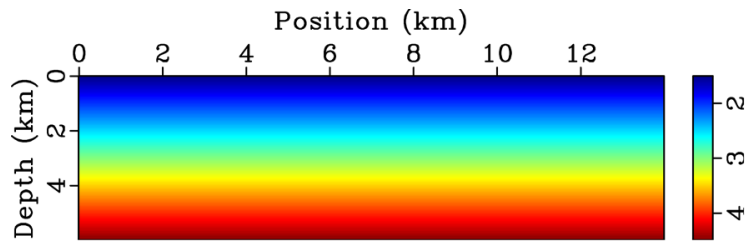

(a)

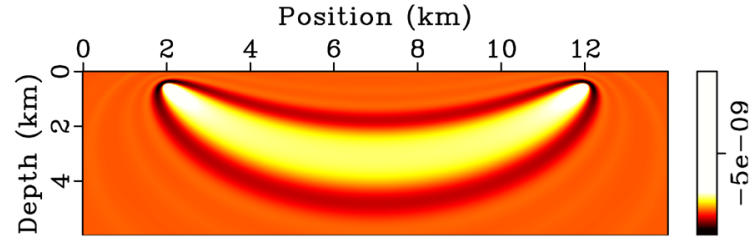

(c)

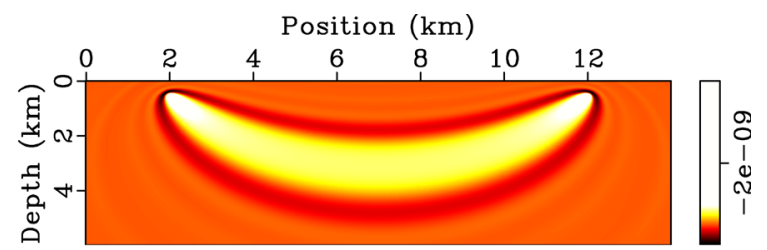

(b)

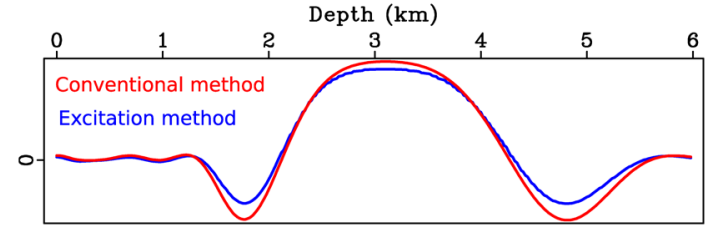

(d)

Figure 4. (a) Linear $v(z)$ model; banana doughnut using (b) ExA and (c) conventional method; (d) depth profile at $x=7.0 \mathrm{~km}$.

by

$\tilde{g}_{\mathrm{ex}}(\mathbf{x})=\frac{\mathbf{v}\left(\mathbf{x}, t_{\mathrm{ex}}(\mathbf{x})\right)}{\mathbf{u}_{\delta}\left(\mathbf{x}, t_{\mathrm{ex}}(\mathbf{x})\right)}$,

which is very similar to the excitation amplitude imaging condition (Nguyen \& McMechan 2013), with a subtle difference in the respective adjoint sources. Nguyen \& McMechan (2013) back propagate the data, whereas we back propagate $\Delta \hat{d}$ (the data-residual cross-correlated with the source wavelet). Comparing with conventional FWI, the excitation approach scales a different wavelet. The former scales down the zero lag cross-correlated $\Delta d$ with source wavefield by the total energy of the source wavefield. On the other 


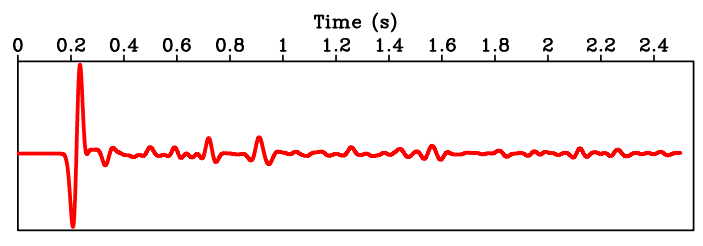

(a)

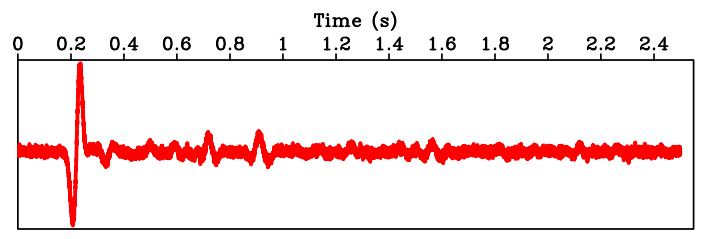

(c)

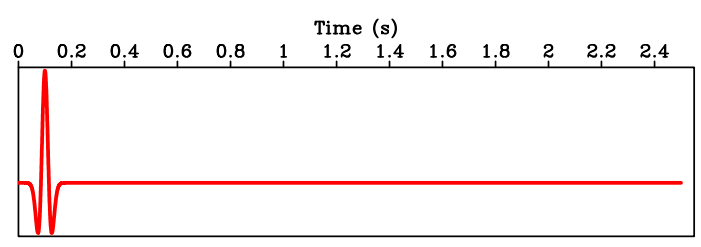

(b)

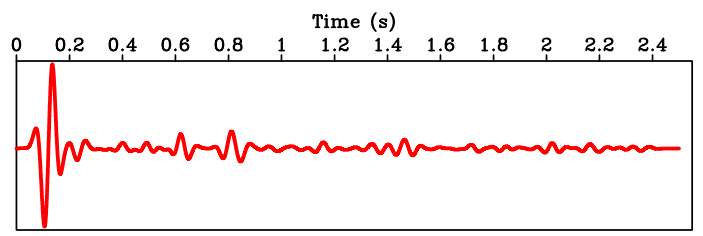

(d)

Figure 5. (a) A seismic trace from the benchmark data of Marmousi model; (b) source wavelet (Ricker) with a dominant frequency of $15 \mathrm{~Hz}$; (c) noisy trace with Gaussian white noise; (d) after cross-correlating noisy trace with the source wavelet.

hand, the excitation approach scales down $\Delta \hat{d}$ by the maximum amplitude of the source wavefield.

\subsection{Insights into the adjoint source of ExA}

Eq. (10) reveals that the source side term $\mathbf{u}_{\delta}$ in the gradient does not have the source signature in it. Therefore, the adjoint source also needs to be deconvolved from the source signature, which is partially accomplished by the temporal cross-correlation process (equivalent to deconvolution in terms of phase) of the source wavelet with the conventional adjoint source. Also, this adjoint source compensates the inversion process for the ignorance of source wavefield's wavelength during the ExA assumption. In addition, the cross-correlation process allows $\Delta \hat{d}$ to have better signal to noise ratio than $\Delta d$, thus results in cleaner gradients, which conventional FWI achieves during the imaging step. In fact, the new adjoint source takes the edge off noise propagation of the data set during the backward extrapolation process. Fig. 5 depicts the improvements in signal to noise ratio in a noisy data by the temporal cross-correlation process with its corresponding source wavelet. We consider a seismic trace from the benchmark data set of Marmousi model (Fig. 5a) and its corresponding source wavelet (Fig. 5b), which is a Ricker wavelet with dominant frequency of $15 \mathrm{~Hz}$. Next, we synthesize a noisy trace as shown in Fig. 5(c) by adding a Gaussian white noise with variance 0.2 to the original trace. Fig. 5(d) displays the retrieved trace with better signal to noise ratio compared to Fig. 5(c). However as described in one of the preceding sections, the signal expectedly suffers a shift in time due to the minimum phase nature of the source wavelet, which will be compensated in the excitation time of the forward propagated signal.

\subsection{Analogy of Kirchhoff algorithm with ExA}

The virtual source for the scattered wavefield with ExA (eqs 5 and 7) resembles a Kirchhoff implementation for the source side, which is represented by $A\left(\mathbf{x} ; \mathbf{x}_{s}\right) \frac{\partial^{2}}{\partial t^{2}} f\left(t-\tau\left(\mathbf{x} ; \mathbf{x}_{s}\right)\right)$, where $A\left(\mathbf{x} ; \mathbf{x}_{s}\right)$ and $\tau\left(\mathbf{x} ; \mathbf{x}_{s}\right)$ are the amplitude and traveltime, respectively. The time, $\tau$ is analogous to $t_{\text {ex }}$ and $A(\mathbf{x})$ to $G_{0}\left(\mathbf{x}, t_{\text {ex }}\right)$, with a difference owing to the band-limited nature of the source function. However, the excitation representation of the source wavefield is immune to the well-known shortcomings of the Kirchhoff methods such as low illumination in the shadow zone or the infinite amplitude at the caustics, as it is calculated using wavefields.

\subsection{Multipathing: a shortcoming of ExA}

The excitation method is more attractive to large data sets, especially in 3-D problems, because of its computational advantages over the conventional FWI. However, its main weakness is handling multipathing in the source wavefield. It is intrinsically handled in conventional FWI by utilizing the full source wavefield with all its complexity. However, an intermediate solution of storing more than one maximum of the source wavefield also can ameliorate the excitation method in the presence of multipathing (Jin et al. 2015; Nguyen \& McMechan 2015). In addition, the initial iterations of FWI are often conducted using smooth (causing multipathing free wavefields) velocity models.

\subsection{Algorithm of FWI with ExA}

The main novelty of this study is computation of gradient in FWI using a single, but the most energetic arrival of the source wavefield. The algorithm can be divided into the following steps:

(i) Start with a good initial velocity $v_{0}$.

(ii) Determine the excitation time of the source wavelet $\left(t_{\mathrm{ex}}^{\mathrm{wlt}}\right)$.

(iii) Forward propagation of the source wavefield: The goal of this step is to compute the excitation time $\left(t_{\mathrm{ex}}\right)$ and amplitude maps of the model. At each time step of the forward propagation, we update the excitation amplitude and time for each grid point in the model only if there is an amplitude increase. Also, we synthesize the model data.

(iv) Time shift correction: $t_{\mathrm{ex}}=t_{\mathrm{ex}}-t_{\mathrm{ex}}^{\mathrm{wlt}}$.

(v) Adjoint source correction: Update the adjoint source by crosscorrelating it with the source wavelet.

(vi) Backward propagation of adjoint wavefield: The goal of this step is to compute the gradient $g_{\text {ex }}$. At each time step of the backward propagation, we perform the imaging step only at the grid points that satisfy the imaging time $\left(t_{\mathrm{ex}}\right)$.

(vii) Step length determination $(\alpha)$.

(viii) Update the background model: $v_{0}=v_{0}-\alpha g_{\text {ex }}$.

(ix) Go back to step (iii) until the termination criteria is satisfied. 


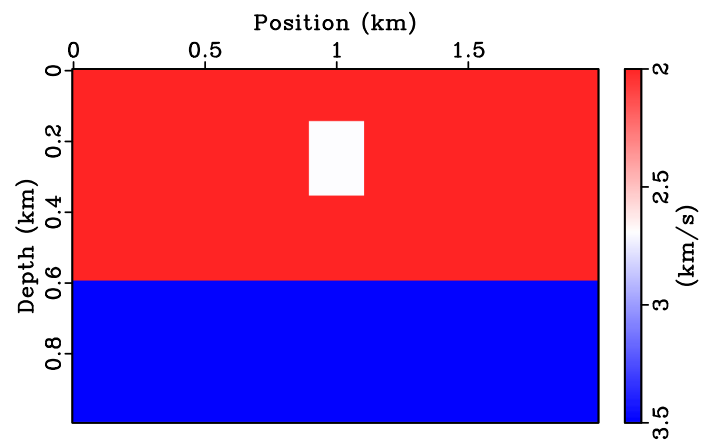

(a)

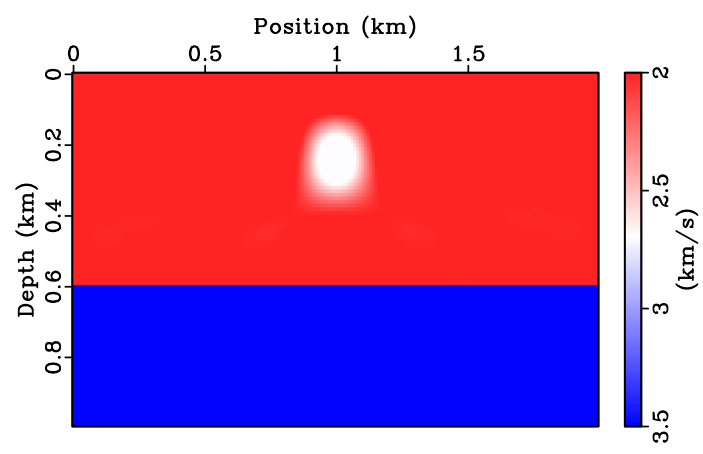

(c)

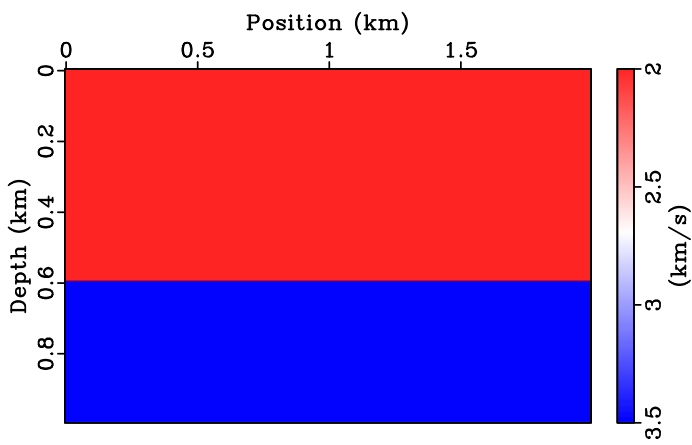

(b)

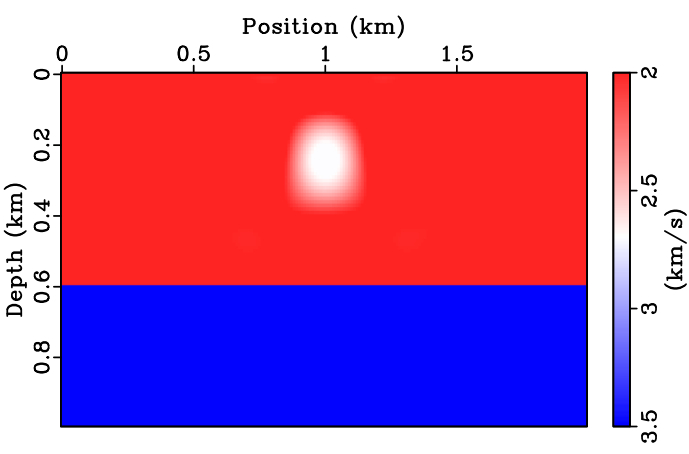

(d)

Figure 6. The panel depicts (a) a two-layer velocity model with a high velocity anomaly, (b) the starting velocity model for inversion; the update after 50 iterations with (c) the excitation approach, and (d) the conventional approach.

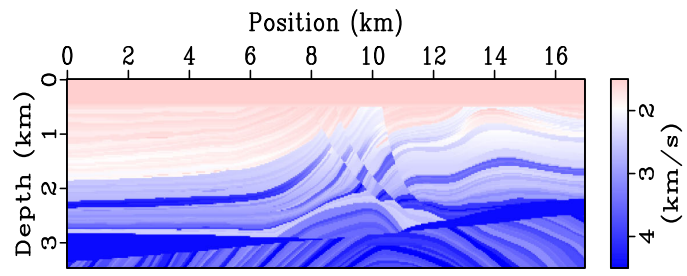

(a)

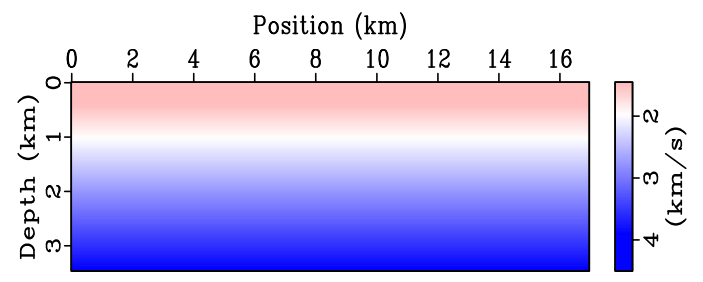

(b)

Figure 7. (a) Marmousi II model; (b) the starting model.

\section{EXAMPLES}

We demonstrate the versatility of the excitation method in FWI on a two-layer velocity model with an anomaly, the Marmousi II model and a real data set. The observed data for the synthetic tests is simulated using the finite difference method, with a second- and fourth-order accuracy in time and space, respectively.

\subsection{A two-layer model with an anomaly}

The high velocity lens anomaly is centred at $(1 \mathrm{~km}, 0.25 \mathrm{~km})$ as shown in Fig. 6(a). We use a Ricker source wavelet with a peak frequency of $2 \mathrm{~Hz}$. Fig. 6(b) displays the initial velocity model. To make the inversion process much easier, we put constraints on both the upper and lower values of velocity in the update process. Also, the update is done only in the first layer of the model. Figs 6(c) and (d) display the inverted velocity after 50 iterations with the excitation method and the conventional approach, respectively. They suggest a good reconstruction of the anomaly in the inversion process. The

update over iterations using the excitation method is similar to its conventional counterpart.

\subsection{Marmousi II model}

The velocity model (Fig. 7a) contains 679 and 139 grid points in $x$ and $z$ direction, respectively. We place 160 sources and 679 receivers on the surface to record the data set. Also, we follow a hierarchal multi-scale strategy Bunks et al. (1995) to invert for two sub data sets of 2 and $4 \mathrm{~Hz}$ dominant frequency, successively. Fig. 7(b) displays the initial velocity model (linearly increasing with depth beneath the water layer). The velocity model after 150 iterations (Fig. 8a) is used as an initial model for the 2 nd stage. The final update is shown in Fig. 8(b). To compare, we display the associated results with that from the conventional approach in Figs 9(a) and (b). A closer look on the depth profiles at $x=8 \mathrm{~km}$ (Fig. 10a) and $x=12 \mathrm{~km}$ (Fig. 10b) reveals some subtle differences in the update between the excitation method and its conventional counterpart. It is due to, most probably, 


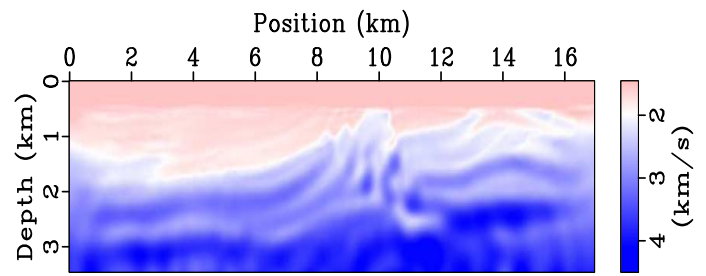

(a)

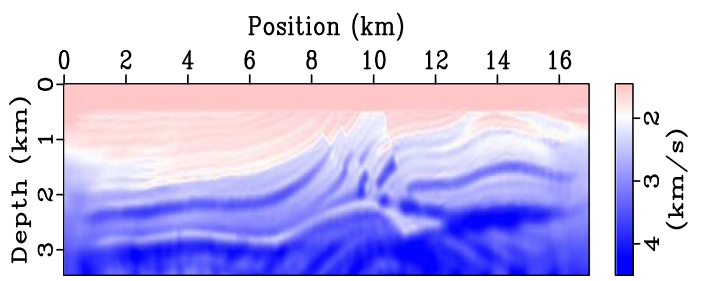

(b)

Figure 8. The velocity update after (a) 150 iterations and (b) 300 iterations using excitation method.

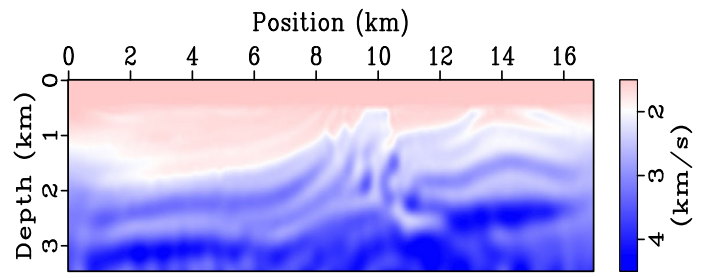

(a)

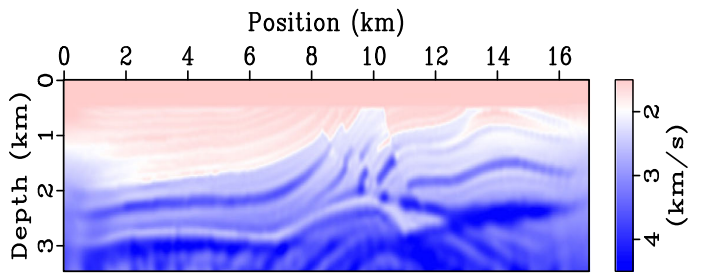

(b)

Figure 9. The velocity update after (a) 150 iterations and (b) 300 iterations using regular FWI.

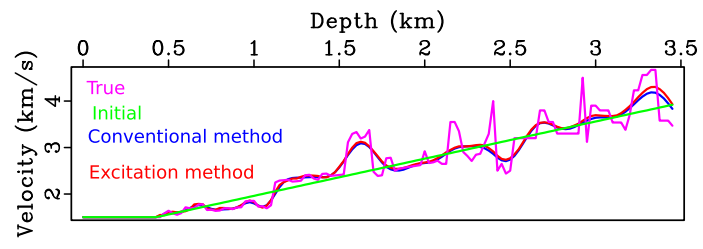

(a)

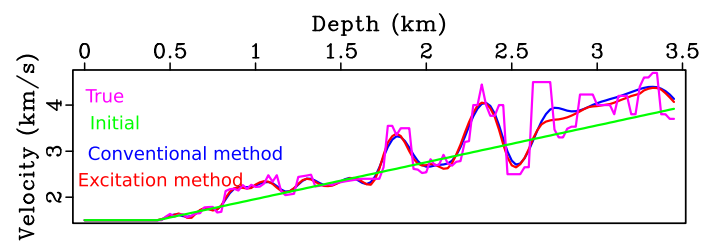

(b)

Figure 10. Comparisons of the depth profiles at (a) $x=8.0 \mathrm{~km}$ and (b) $x=12.0 \mathrm{~km}$ for the two methods.

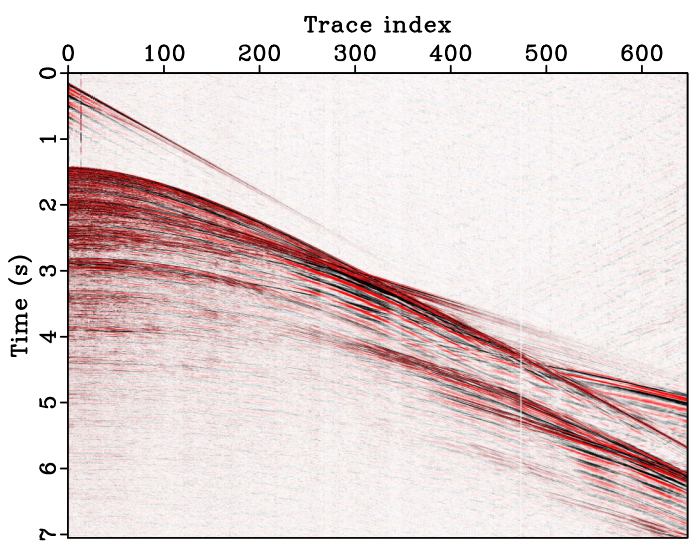

(a)

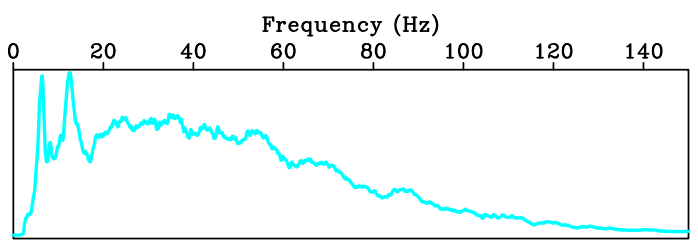

(b)

Figure 11. (a) Shot gather with the source at $(x, z)=(1.875,0.005) \mathrm{km}$ and (b) its average frequency spectrum.

the loss of some multipath energy from the source side, a weakness of the excitation approach.

\subsection{Real data set}

This example contains a 2-D marine data set from North-Western Australia Continental Shelf, acquired by CGG using a Broadseis acquisition system with a variable depth streamer (Soubaras \& Dowle 2010). This kind of acquisition technique enhances the low frequency content of the recorded data. CGG has filtered out data with frequencies below $2.5 \mathrm{~Hz}$ because of the poor signal-to-noise ratio.
Fig. 11 displays one shot gather and its average frequency content. Although the original data set is composed of 1824 shot gathers with an interval of approximately $0.01875 \mathrm{~km}$, we choose the first 576 shot gathers corresponding to the reduced area of investigation in our inversion process. Each streamer contains 648 receivers with a group interval of approximately $0.0125 \mathrm{~km}$. The near and far offset are approximately 0.169 and $8.3 \mathrm{~km}$, respectively. Next, we choose the velocity model as $12.5 \mathrm{~km}$ long in $x$ direction and $3.75 \mathrm{~km}$ deep in $z$ direction. Fig. 12 displays the number of traces per shot bounded by the physical domain of the model under consideration. Also, we show the average frequency content of the shot gather (Fig. 11a) at 


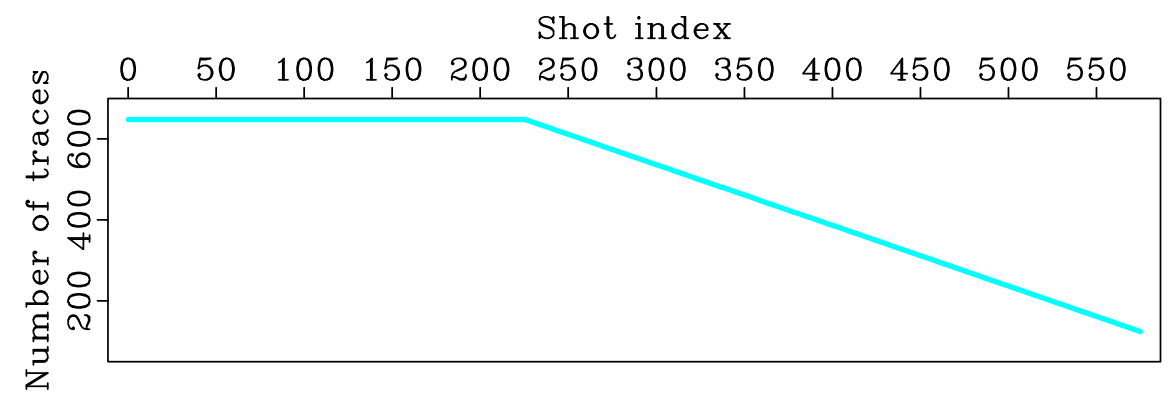

Figure 12. Number of traces per shot.

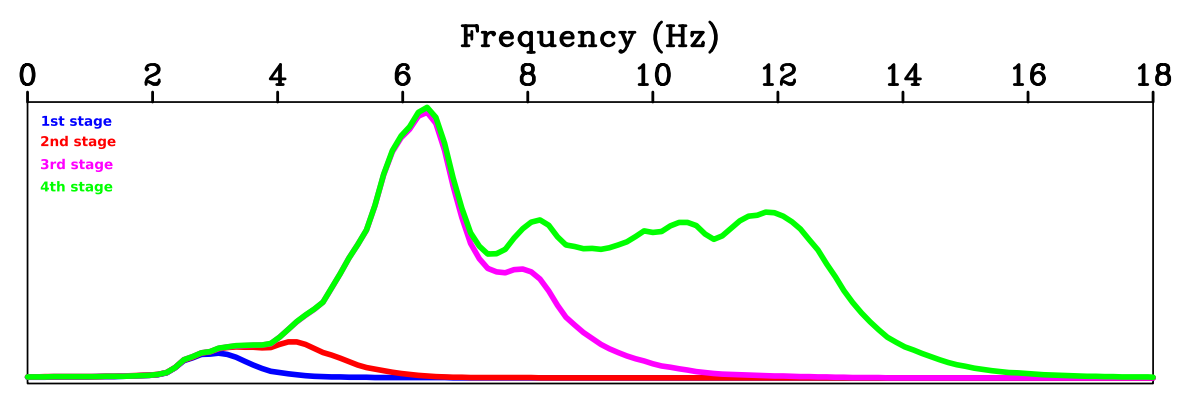

Figure 13. Average frequency content of the shot gather shown in Fig. 14(a) at different stages of the hierarchal approach.

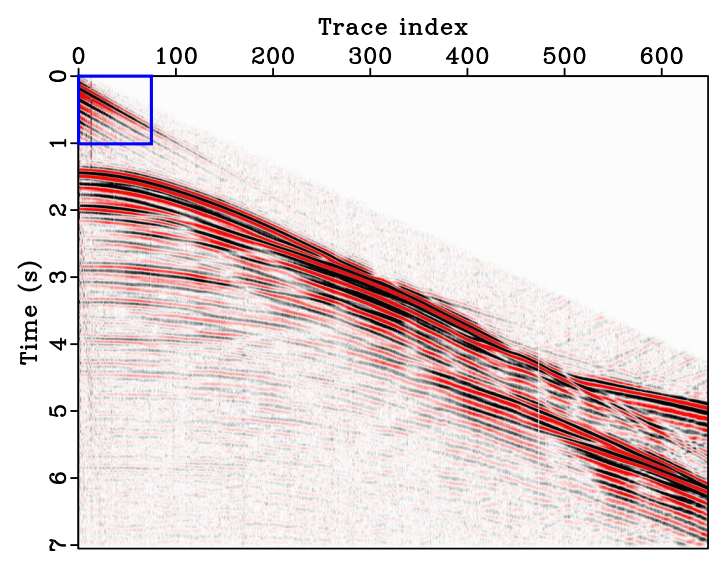

(a)

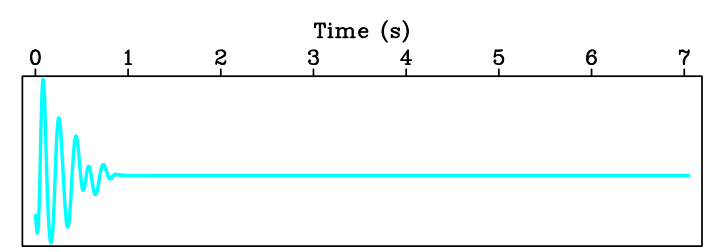

(b)

Figure 14. Seismic traces in the blue box of (a) is considered to invert for the source wavelet (b).

different stages of the hierarchal multi-scale approach (Bunks et al. $1995)$ in Fig. 13. Before the inversion process starts, we execute the steps below:

(i) Processing steps. We mute the noise before the first arrival.

(ii) Source wavelet estimation. The method adapted to do so is described in Kim et al. (2011), which requires the portion of seismic traces with the known velocity. We consider the direct arrivals of some near-offset traces, for instance marked in the blue box of Fig. 14 (a), assuming the water velocity to be $v_{\text {water }}=1.49 \mathrm{~km} \mathrm{~s}^{-1}$. Fig. 14(b) shows the corresponding inverted source wavelet.

(iii) Initial velocity model estimation. We rely on a velocity obtained using a migration velocity analysis technique, specifically reflection waveform inversion (Wu \& Alkhalifah 2015a,b), shown in Fig. 15. Next, we pick the water bottom of the RTM image (Fig. 16) of the data, migrated with $v_{\text {water }}$ and keep the water layer velocity unchanged throughout the inversion process.

(iv) Objective function (J). We choose $J$ as suggested by Choi \& Alkhalifah (2012), which is the zero lag cross-correlation between the normalized modelled $\left(\tilde{d}_{m}=d_{m} /\left\|d_{m}\right\|\right)$ and normalized observed data $\left(\tilde{d}_{o}=d_{o} /\left\|d_{o}\right\|\right)$ :

$J=-\left\langle\tilde{d}_{m}, \tilde{d}_{o}\right\rangle$

where || || represents the norm of the wavefield. Accordingly, the gradient evaluation of this objective function is obtained using eq. (11), but with a modification in the adjoint source given by:

$\Delta \hat{d}=\frac{1}{\left\|d_{m}\right\|} \mathbf{F}^{*}\left\{\left\langle\tilde{d}_{m}, \tilde{d}_{o}\right\rangle \tilde{d}_{m}-\tilde{d}_{o}\right\}$.

We display the evolution of the velocity model during the course of the inversion process in Fig. 17. It gradually enriches the initial model with high wavenumber components of velocity. Moreover, we overlay an available $\log$ profile from a borehole at $x \approx 10.6 \mathrm{~km}$ on the final velocity model of our inversion. The log suggests well resemblance $\left(\mathrm{rms}\right.$ error $=0.3 \mathrm{~km} \mathrm{~s}^{-1}$ ) with the corresponding depth profile of the velocity model. As expected, the objective function also decreases over iterations (Fig. 18). 


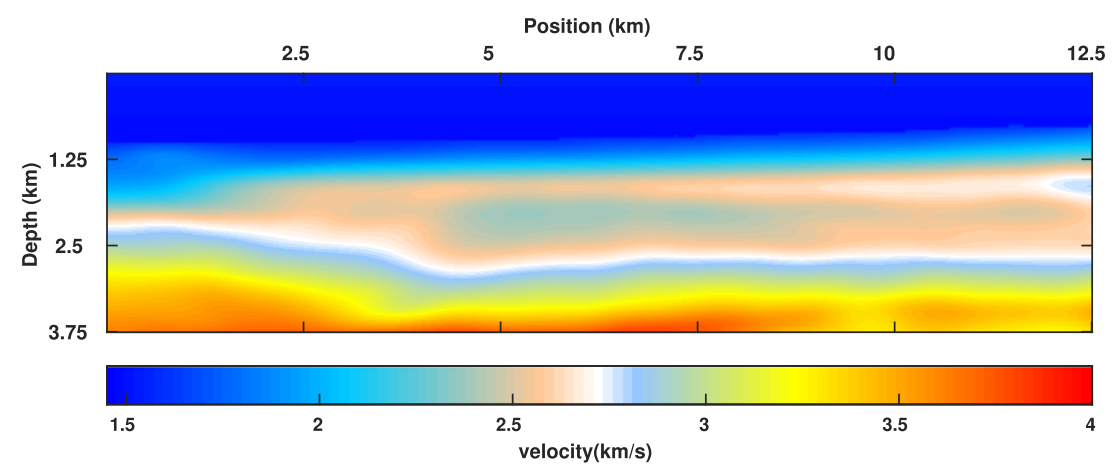

Figure 15. Initial velocity model.

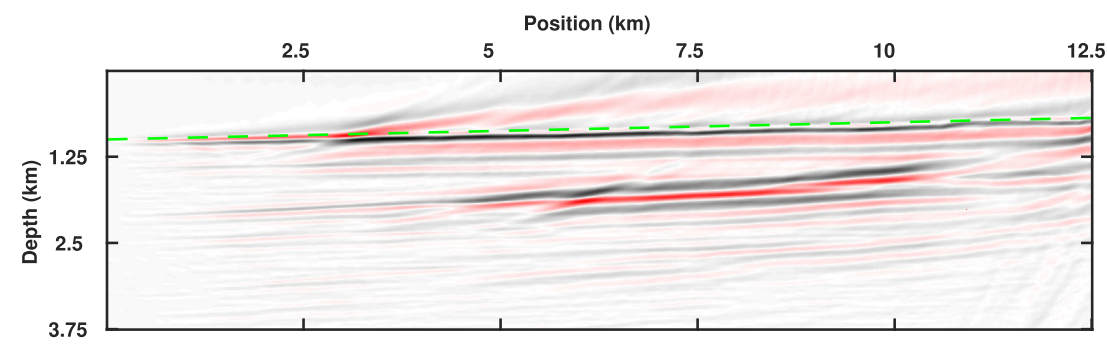

Figure 16. RTM image with $v_{\text {water }}$ as migration velocity. Green line indicates the sea-bottom.

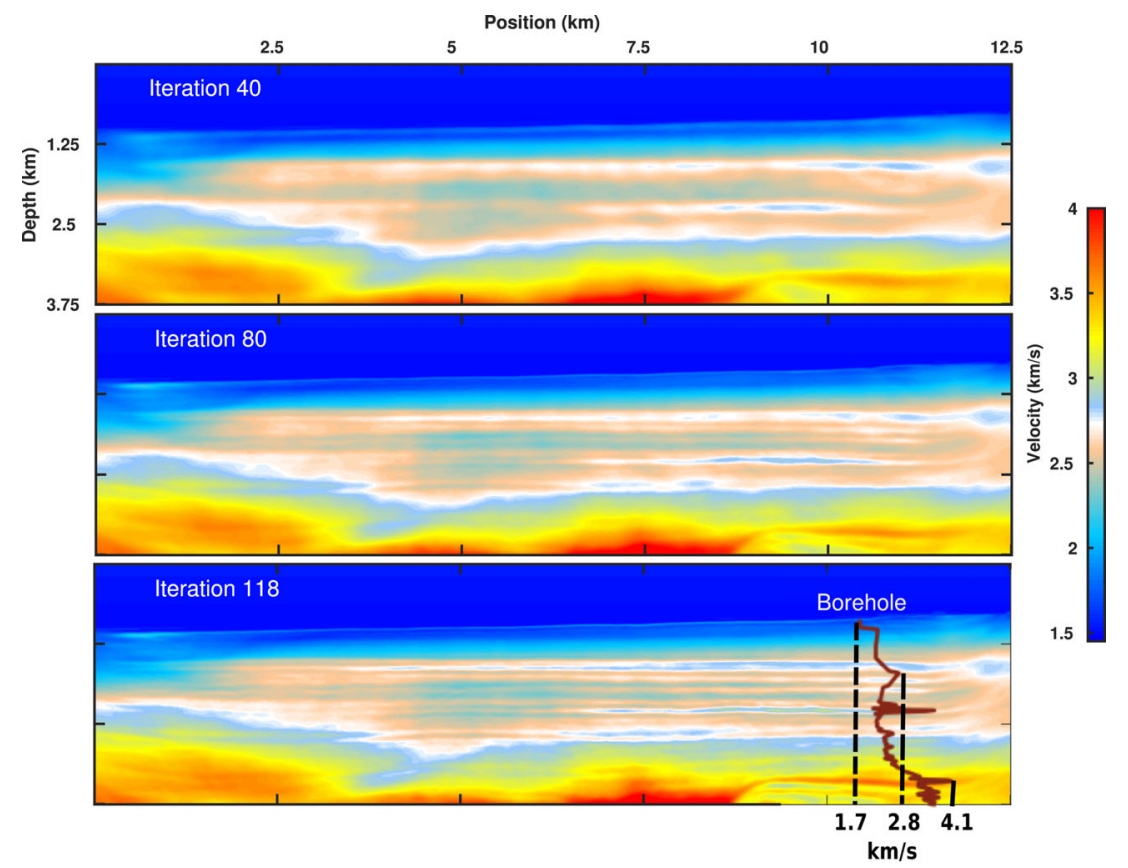

Figure 17. Velocity model at iteration $\{40,80,118\}$. The available log data of the borehole in the bottom panel follow a similar trend of the velocity depth profile with an rms error of $0.3 \mathrm{~km} \mathrm{~s}^{-1}$.

We display the final velocity model using the conventional approach in Fig. 19, which is almost identical to its excitation counterpart. However, the excitation method managed to reduce the computational cost and memory requirements, as discussed in Section 3.4. Next, we compare $d_{o}$ (Fig. 14a) with $d_{m}$ from the initial and final inverted velocity models in Fig. 20. To make this comparison more quantitative, we plot the correlation factor (C.F.), defined as (Wu \& Alkhalifah 2015b):

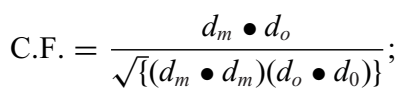

in Fig. 21(b). Two sets of identical signals yield a C.F. of unity. The inversion process has certainly improved our initial guess as indicated by the C.F. in Fig. 21(b). Next, we compute the angle domain common-image gathers (ADCIGs) at $x=\{2,3,4,5,6$, $7,8,9\} \mathrm{km}$ (Fig. 22) using an efficient method based on excitation approach (Kalita \& Alkhalifah 2016). Compared to conventional approach, this method (eq. 16) computes the subsurfaceoffset common-image gathers $\left(I_{\mathrm{ex}}(\mathbf{x}, \mathbf{h})\right)$ with a requirement of much shorter computational time and lower memory/hard disk:

$I_{\mathrm{ex}}(\mathbf{x}, \mathbf{h})=\frac{\overleftarrow{d_{o}}\left(\mathbf{x}+\mathbf{h}, t_{\mathrm{ex}}(\mathbf{x}-\mathbf{h})\right)}{G_{0_{\mathrm{ex}}}\left(\mathbf{x}-\mathbf{h}, t_{\mathrm{ex}}(\mathbf{x}-\mathbf{h})\right)}$, 


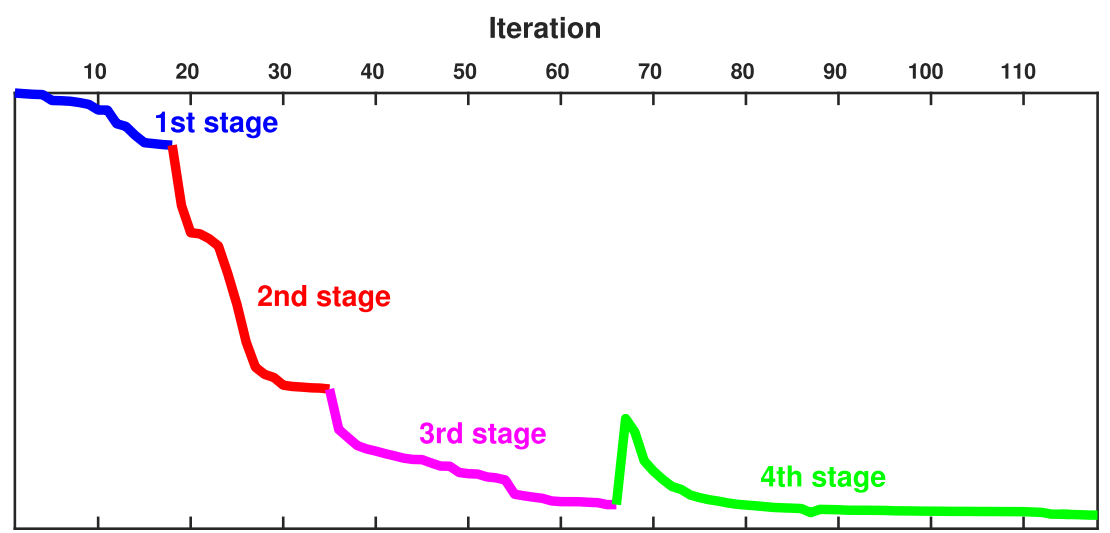

Figure 18. Objective function decreases over iterations.

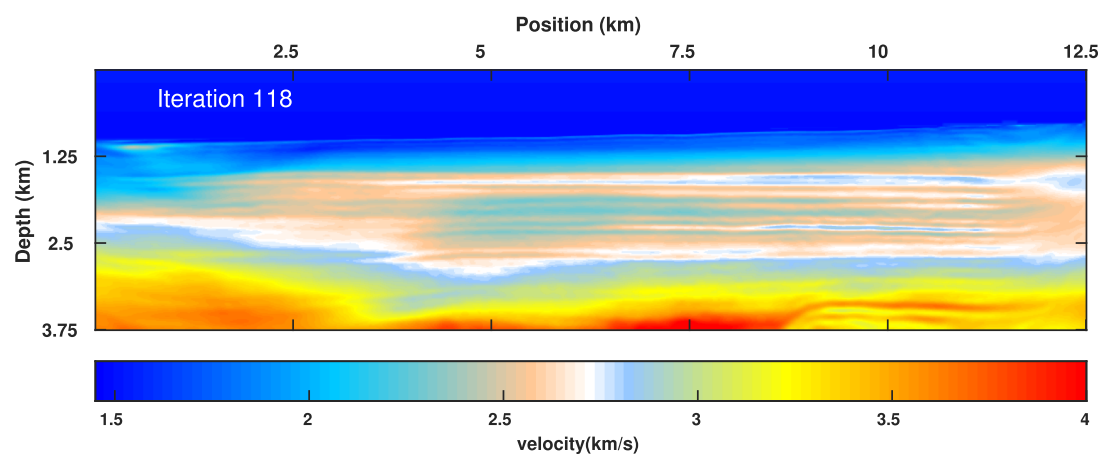

Figure 19. Final velocity model using conventional FWI.

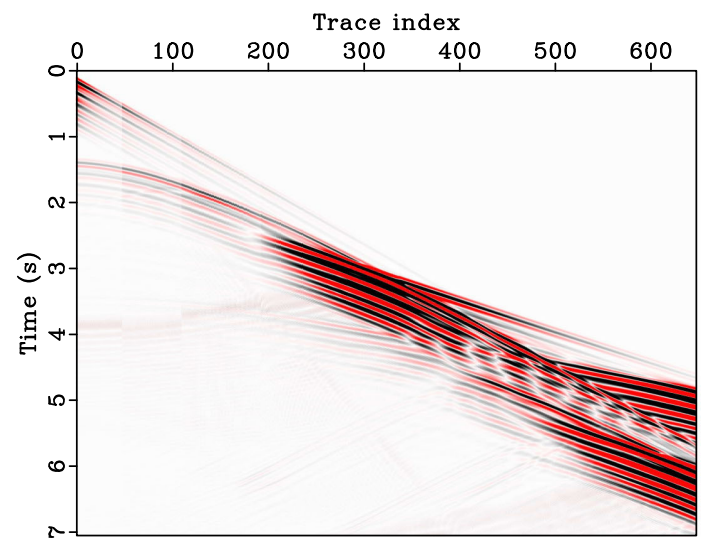

(a)

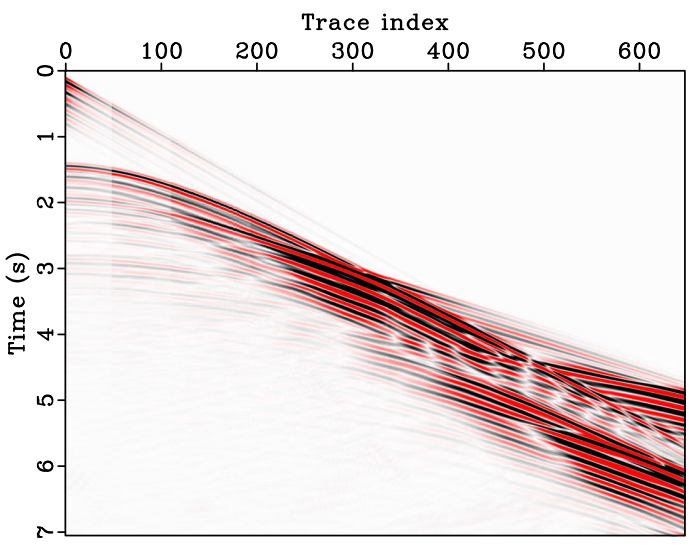

(b)

Figure 20. Modelled data with (a) initial velocity and (b) final velocity. Compare with Fig. 14(a).

where $\mathbf{h}$ represents the half sub-surface offset. Conversion to ADCIGs thereafter follows the regular method. As shown in Fig. 22(a), ADCIGs using the initial velocity are already flat, especially at shallow depths, validating the fact that the initial model predicted the kinematics accurately. After inversion, the final velocity model maintained the ADCIGs accuracy (Fig. 22b). In addition, we plot the RTM image with excitation amplitude imaging condition (Nguyen \& McMechan 2013) using the initial and final velocity model in Fig. 23. We observe a subtle difference in focusing of the reflectors, especially around $z=1.75 \mathrm{~km}$, inside the blue box of the bottom panel in Fig. 23. They are more focused with final velocity model compared with the initial.

\subsection{Computational requirements}

FWI with excitation approach is superior to that of the conventional approach in terms of computational overhead. Since there is no requirement of storing the full history of the source wavefield, it computes the gradient accessing a very limited memory block. Also, it avoids the redundant modelling step that is required to retrieve the source wavefield from the boundary values, thus, results in faster evaluation of the gradient. Tables 1 and 2 display the computational cost and memory involved in computing the gradient for one shot gather of the examples presented in Section 3. It reveals the following important points: 


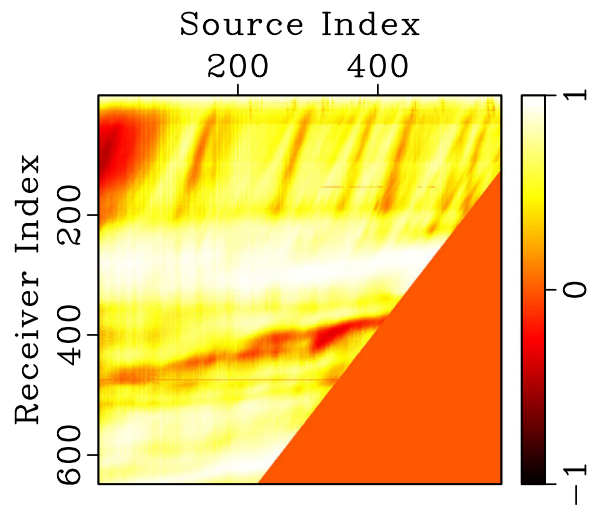

(a)

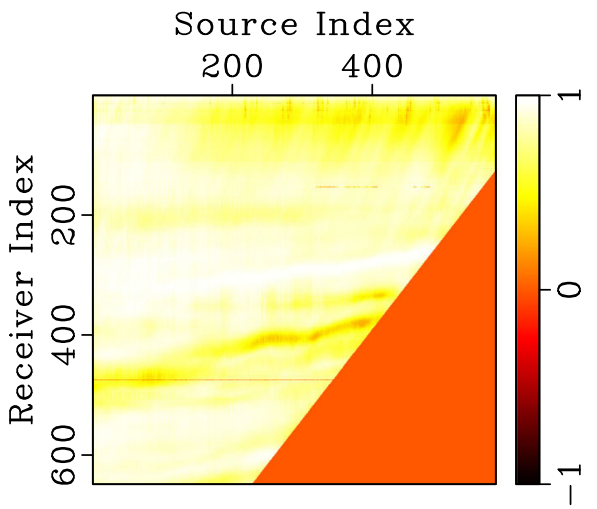

(b)

Figure 21. Correlation factor associated with all the traces in the data set using the (a) initial velocity and (b) final velocity model. Note that the zero values in the right corner of the maps are because of the unavailability of traces (refer Fig. 12).

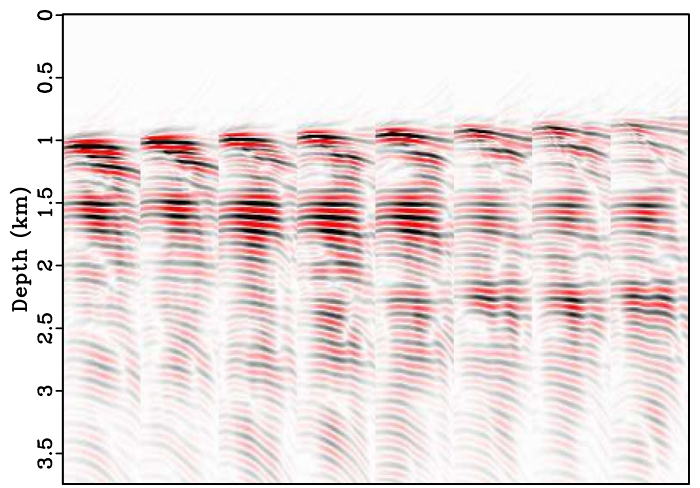

(a)

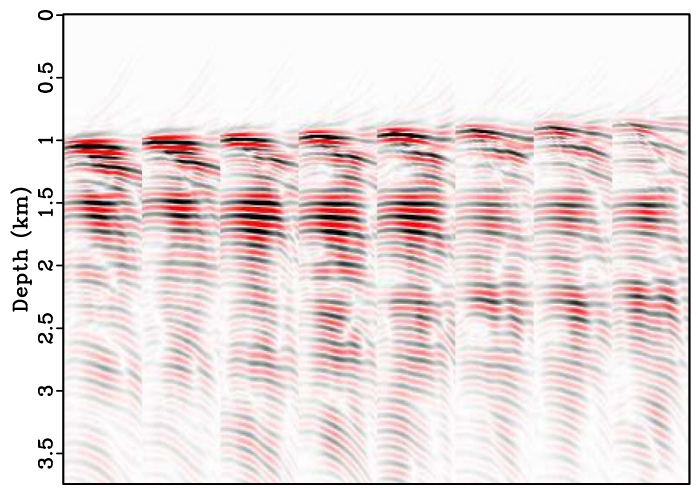

(b)

Figure 22. ADCIGs at $x=\{2,3,4,5,6,7,8,9\} \mathrm{km}$ using (a) initial velocity and (b) final velocity.
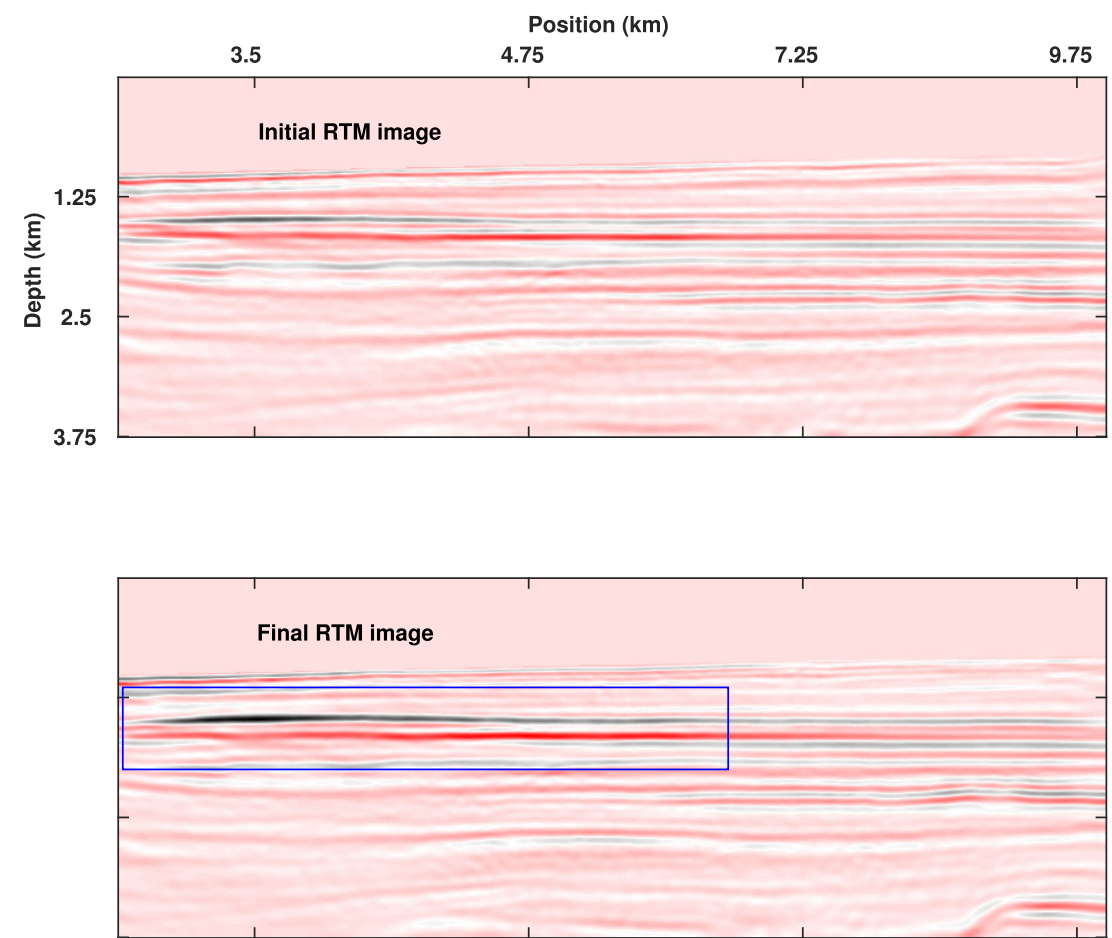

Figure 23. Top panel depicts RTM image with the initial velocity model and the bottom panel with the final velocity model. The events inside the green box are more focused compared to the corresponding events in the top panel. 
Table 1. Required computational time to evaluate the gradient for one shot gather.

\begin{tabular}{lcccc}
\hline & Model size & Data size & $\begin{array}{c}\text { Conventional method } \\
\text { (boundary saving) }\end{array}$ & $\begin{array}{c}\text { Conventional method } \\
\text { (history saving) }\end{array}$ \\
\hline Section 3.1 & $100 * 200$ & $2000 * 200$ & $7 \mathrm{~s}$ & $4 \mathrm{~s}$ \\
Section 3.1 & $139 * 679$ & $4500 * 679$ & $65 \mathrm{~s}$ & $41 \mathrm{~s}$ \\
Section 3.3 & $300 * 1000$ & $7052 * 648$ & $110 \mathrm{~s}$ & $72 \mathrm{~s}$ \\
\hline
\end{tabular}

Table 2. Computational memory usage to compute the gradient for one shot gather.

\begin{tabular}{lcccr}
\hline & Dodel size & Data size & $\begin{array}{c}\text { Conventional method } \\
\text { (boundary saving scheme) }\end{array}$ & \multicolumn{2}{c}{$\begin{array}{c}\text { Conventional method } \\
\text { (history saving scheme) }\end{array}$} \\
\hline Section 3.1 & $100 * 200$ & $2000 * 200$ & $24 \mathrm{MB}$ & $172 \mathrm{MB}$ \\
Section 3.1 & $139 * 679$ & $4500 * 679$ & $100 \mathrm{MB}$ & $1 \mathrm{~GB} 727 \mathrm{MB}$ \\
Section 3.3 & $300 * 1000$ & $7052 * 648$ & $226 \mathrm{MB}$ & $44 \mathrm{MB}$ \\
\hline
\end{tabular}

(i) ExA invests slightly longer computational time than the full history saving scheme in the process of evaluating the gradient. This inefficiency occurs primarily because of two additional steps involved in the algorithm (Section 2.8): (1) computation of excitation maps and (2) determination of adjoint source which requires a temporal cross-correlation process. However, this additional cost of ExA is negligible considering the requirements of limited memory size in order to compute the gradient. As expected, ExA is computationally faster than the boundary saving scheme, often used in conventional FWI for large 3-D problems.

(ii) ExA requires the lowest computational memory amongst all. It consumes a memory block of the order of the model size, which provides the method the upper hand over conventional FWI in large $3-\mathrm{D}$ problems. Although promising in 2-D, the boundary saving scheme suffers from memory issue in large 3-D problems (Yang et al. 2016).

\section{DISCUSSION}

The excitation approach utilizes the most energetic part of the source wavefield in order to compute the gradient. In the Marmousi example, where multipathing is prevalent, such limitation had minor effect on the quality of the inversion. Most of the energy to the gradient computation is usually extracted from a single scattering optimal (energy wise) path of the waves. In some cases, mutipathing must be addressed accurately to have a successful inversion.

Alternatively, we can have even a more efficient implementation by extracting the source wavefield excitation information from solving the eikonal equation or ray tracing (Chang \& McMechan 1986; Nichols 1996), but at the cost of reduced accuracy as we confine ourselves to the high-frequency asymptotic approximation and its limitations. Despite the high-frequency asymptotic nature of such approximations, the often simple nature of the source wavefield, compared to the receiver wavefield, allows us to use such approximations, especially at the early iterations of FWI where the model is still generally smooth.

\section{CONCLUSIONS}

We develop Born modelling and FWI based on the excitation representation of the source wavefield. It, specifically, simplifies the source wavefield by the maximum amplitude and its arrival time, and thus, reduces the enormous storage requirements for the source wavefield in the conventional implementation of FWI. Gradient evaluation is done only at the time of excitation, no dot product is needed, resulting in a reduction of the number of FLOPs for such computation. Although, multipathing is a shortcoming of this method, such limitation had minor effects in the inversion process, especially in the early iterations, as observed in the inversion of Marmousi II model and the real data set.

\section{ACKNOWLEDGEMENTS}

For computer time, this research used the resources of the Supercomputing Laboratory and IT Research Computing at King Abdullah University of Science \& Technology (KAUST) in Thuwal, Saudi Arabia. The real data shown in this study are proprietary to and provided courtesy of CGG. The well-log information is provided by Geoscience Australia. We are grateful to KAUST for financial support and all the members of seismic wave analysis group (SWAG), especially Christos Tzivanakis, Zedong Wu, Juwon Oh, Yunseok Choi and Vladimir Kazei for their fruitful discussions. In addition, we are especially thankful to Zedong $\mathrm{Wu}$ for his assistance in the real data example.

\section{REFERENCES}

Albertin, U., Shan, G. \& Washbourne, J., 2013. Gradient orthogonalization in adjoint scattering-series inversion, in 2013 SEG Annual Meeting, Society of Exploration Geophysicists.

Alkhalifah, T., 2015. Scattering-angle based filtering of the waveform inversion gradients, Geophys. J. Int., 200(1), 363-373.

Alkhalifah, T. \& Wu, Z., 2016. The natural combination of full and imagebased waveform inversion, Geophys. Prospect., 64(1), 19-30.

Allemand, T. \& Lambaré, G., 2015. Combining full waveform inversion and tomography: full waveform inversion-guided tomography, in 77th EAGE Conference and Exhibition 2015, EAGE.

Almomin, A. \& Biondi, B., 2012. Tomographic full waveform inversion: practical and computationally feasible approach, in 2012 SEG Annual Meeting, Society of Exploration Geophysicists.

Berkhout, A.J., 1988. A holographic approach to acoustic control, J. Audio Eng. Soc., 36(12), 977-995.

Biondi, B. \& Almomin, A., 2014. Simultaneous inversion of full data bandwidth by tomographic full-waveform inversion, Geophysics, 79(3), WA129-WA140.

Boehm, C., Hanzich, M., de la Puente, J. \& Fichtner, A., 2016. Wavefield compression for adjoint methods in full-waveform inversion, Geophysics, 81(6), R385-R397.

Boonyasiriwat, C., Valasek, P., Routh, P., Cao, W., Schuster, G.T. \& Macy, B., 2009. An efficient multiscale method for time-domain waveform tomography, Geophysics, 74(6), WCC59-WCC68.

Bunks, C., Saleck, F.M., Zaleski, S. \& Chavent, G., 1995. Multiscale seismic waveform inversion, Geophysics, 60(5), 1457-1473. 
Chang, W.-F. \& McMechan, G.A., 1986. Reverse-time migration of offset vertical seismic profiling data using the excitation-time imaging condition, Geophysics, 51(1), 67-84.

Choi, Y. \& Alkhalifah, T., 2011. Source-independent time-domain waveform inversion using convolved wavefields: application to the encoded multisource waveform inversion, Geophysics, 76(5), R125-R134.

Choi, Y. \& Alkhalifah, T., 2012. Application of multi-source waveform inversion to marine streamer data using the global correlation norm, Geophys. Prospect., 60(4), 748-758.

Clapp, R.G., 2008. Reverse time migration: saving the boundaries, Stanford Exploration Project, p. 136.

Dalmau, F.R., Hanzich, M., de la Puente, J. \& Gutiérrez, N., 2014. Lossy data compression with DCT transforms, in EAGE Workshop on High Performance Computing for Upstream, Crete, Greece.

Djebbi, R. \& Alkhalifah, T., 2013. Finite frequency traveltime sensitivity kernels for acoustic anisotropic media: angle dependent bananas, in 2013 SEG Annual Meeting, Society of Exploration Geophysicists.

Fichtner, A., Trampert, J., Cupillard, P., Saygin, E., Taymaz, T., Capdeville, Y. \& Villaseñor, A., 2013. Multiscale full waveform inversion, Geophys. J. Int., 194, 534-556.

Gauthier, O., Virieux, J. \& Tarantola, A., 1986. Two-dimensional nonlinear inversion of seismic waveforms: numerical results, Geophysics, 51(7), 1387-1403.

Jin, H., McMechan, G.A. \& Nguyen, B., 2015. Improving input/output performance in $2 \mathrm{D}$ and $3 \mathrm{D}$ angle-domain common-image gathers from reverse time migration, Geophysics, 80(2), S65-S77.

Kalita, M. \& Alkhalifah, T., 2016. Common-image gathers using the excitation amplitude imaging condition, Geophysics, 81(4), S261-S269.

Kazei, V., Tessmer, E. \& Alkhalifah, T., 2016. Scattering angle-based filtering via extension in velocity, in SEG Technical Program Expanded Abstracts 2016, pp. 1157-1162, Society of Exploration Geophysicists.

Kim, Y., Min, D.-J. \& Shin, C., 2011. Frequency-domain reverse-time migration with source estimation, Geophysics, 76(2), S41-S49.

Krebs, J.R., Anderson, J.E., Hinkley, D., Neelamani, R., Lee, S., Baumstein, A. \& Lacasse, M.-D., 2009. Fast full-wavefield seismic inversion using encoded sources, Geophysics, 74(6), WCC177-WCC188.

Lailly, P., 1983. The seismic inverse problem as a sequence of before stack migrations, in Conference on Inverse Scattering: Theory and Application, pp. 206-220, SIAM, Philadelphia, PA.

Ma, Y. \& Hale, D., 2012. Quasi-newton full-waveform inversion with a projected hessian matrix, Geophysics, 77(5), R207-R216.

Métivier, L., Bretaudeau, F., Brossier, R., Operto, S. \& Virieux, J., 2014. Full waveform inversion and the truncated newton method: quantitative imaging of complex subsurface structures, Geophys. Prospect., 62(6), 1353-1375.

Mittal, S. \& Vetter, J.S., 2016. A survey of architectural approaches for data compression in cache and main memory systems, IEEE Trans. Parallel Distrib. Syst., 27(5), 1524-1536.

Nguyen, B.D. \& McMechan, G.A., 2013. Excitation amplitude imaging condition for prestack reverse-time migration, Geophysics, 78, S37-S46.

Nguyen, B.D. \& McMechan, G.A., 2015. Five ways to avoid storing source wavefield snapshots in 2D elastic prestack reverse time migration, Geophysics, 80(1), S1-S18.

Nichols, D.E., 1996. Maximum energy traveltimes calculated in the seismic frequency band, Geophysics, 61(1), 253-263.
Nocedal, J. \& Wright, S., 2006. Numerical Optimization, Springer Science \& Business Media.

Plessix, R.-E., 2006. A review of the adjoint-state method for computing the gradient of a functional with geophysical applications, Geophys. J. Int., 167(2), 495-503.

Pratt, R.G., 1990. Inverse theory applied to multi-source cross-hole tomography., Geophys. Prospect., 38(3), 311-329.

Pratt, R.G., Song, Z.-M., Williamson, P. \& Warner, M., 1996. Twodimensional velocity models from wide-angle seismic data by wavefield inversion, Geophys. J. Int., 124(2), 323-340.

Pratt, R.G., Shin, C. \& Hick, G., 1998. Gauss-Newton and full newton methods in frequency-space seismic waveform inversion, Geophys. J. Int., 133(2), 341-362.

Schuster, G.T., Wang, X., Huang, Y., Dai, W. \& Boonyasiriwat, C., 2011. Theory of multisource crosstalk reduction by phase-encoded statics, Geophys. J. Int., 184(3), 1289-1303.

Shin, C., Jang, S. \& Min, D.-J., 2001. Improved amplitude preservation for prestack depth migration by inverse scattering theory, Geophys. Prospect., 49(5), 592-606.

Sirgue, L. \& Pratt, R.G., 2004. Efficient waveform inversion and imaging: a strategy for selecting temporal frequencies, Geophysics, 69(1), 231248.

Soubaras, R. \& Dowle, R., 2010. Variable-depth streamer-a broadband marine solution, First Break, 28(12).

Symes, W.W., 2008. Migration velocity analysis and waveform inversion, Geophys. Prospect, 56(6), 765-790.

Tang, Y., Lee, S., Baumstein, A. \& Hinkley, D., 2013. Tomographically enhanced full wavefield inversion, in 2013 SEG Annual Meeting, Society of Exploration Geophysicists.

Tarantola, A., 1984. Inversion of seismic reflection data in the acoustic approximation, Geophysics, 49(8), 1259-1266.

Tarantola, A., 2005. Inverse Problem Theory and Methods for Model Parameter Estimation, SIAM.

Tromp, J., Tape, C. \& Liu, Q., 2005. Seismic tomography, adjoint methods, time reversal and banana-doughnut kernels, Geophys. J. Int., 160(1), 195216.

Unat, D., Hromadka III, T. \& Baden, S.B., 2009. An adaptive sub-sampling method for in-memory compression of scientific data, in 2009 Data Compression Conference, pp. 262-271, IEEE.

van der Hilst, R.D. \& de Hoop, M.V., 2005. Banana-doughnut kernels and mantle tomography, Geophys. J. Int., 163(3), 956-961.

Virieux, J. \& Operto, S., 2009. An overview of full-waveform inversion in exploration geophysics, Geophysics, 74(6), WCC1WCC26.

Woodward, M.J., 1992. Wave-equation tomography, Geophysics, 57(1), 1526.

Wu, Z.\& Alkhalifah, T., 2015a. Full waveform inversion based on scattering angle enrichment with application to real dataset, in 2015 SEG Annual Meeting, Society of Exploration Geophysicists.

Wu, Z. \& Alkhalifah, T., 2015b. Simultaneous inversion of the background velocity and the perturbation in full-waveform inversion, Geophysics, 80(6), R317-R329.

Yang, P., Brossier, R. \& Virieux, J., 2016. Wavefield reconstruction by interpolating significantly decimated boundaries, Geophysics, 81(5), T197T209. 